\title{
Non-decoupling effects due to a dimensionful coupling
}

\author{
Kenichiro Aoki ${ }^{1}$ \\ Department of Physics, Hiyoshi Campus \\ Keio University \\ 4-1-1 Hiyoshi, Kouhoku-ku \\ Yokohama 223, JAPAN
}

\begin{abstract}
We show that the dimensionful scalar cubic coupling in $3+1$ dimensions gives rise to non-decoupling effects and analyze the behavior of these effects. In the process, we clarify how it is perturbatively consistent to construct theories in which the cubic coupling is dominant. We use the scalar sector of the Supersymmetric Standard Model as an example. We discuss how the non-decoupling effects may be analyzed systematically.
\end{abstract}

1 Email: ken@phys-h.keio.ac.jp 


\section{Introduction}

The properties of particles too heavy to be produced in experiments may sometimes be extracted through their physically observable quantum effects. Under reasonably general conditions, the decoupling theorem [1] applies and these effects become smaller as their masses become larger. However, there also exist so-called "non-decoupling effects" wherein the assumptions of the decoupling theorem are not satisfied. In such a situation, a heavy particle can give rise to physically observable quantum effects that do not necessarily decrease with the particle mass. The importance of these non-decoupling effects hardly needs to be stressed; they were used to obtain a perturbative bound for the top mass [2] which was borne out in experiment [3]. (Non-perturbative considerations are needed for a very heavy top mass, which have been analyzed in [4] [5] [6].) Non-decoupling effects have also been instrumental in the investigations of observable effects of the various extensions of the Standard Model, which have been performed at an impressive level. [7]

While the importance of non-decoupling effects is universally accepted, the properties of non-decoupling effects are not systematically understood. This is to be contrasted to the decoupling effects which are known to decrease as a power of the particle mass, up to logarithms, when we increase the particle mass as we keep the coupling constant fixed [1]. To systematically understand non-decoupling effects, we first need to establish which couplings may give rise to non-decoupling effects. Furthermore, we need to analyze the mass dependence of these effects since it is of crucial importance in determining the actual size of the effects. It is desirable to have a systematic understanding of these effects, wherein the couplings are classified as those that may give rise to non-decoupling effects or those which do not, and if they do give rise to non-decoupling effects, what their possible leading order mass dependence may be. Such a systematic understanding, even perturbatively, is lacking at this point.

It is well-known that the dimensionless couplings, namely the Yukawa and the scalar quartic coupling, give rise to non-decoupling effects. For instance, in the Standard Model, the top and the Higgs contribute to the $\rho$ parameter at the one loop level as

$$
\delta \rho_{\mathrm{top}}=\frac{3}{(4 \pi)^{2}} \sqrt{2} G_{F} m_{t}^{2} \quad, \quad \delta \rho_{\mathrm{Higgs}}=-\frac{3}{4} \frac{g^{\prime 2}}{(4 \pi)^{2}} \log \left(\frac{M_{H}^{2}}{M_{W}^{2}}\right)
$$

Quantum corrections due to gauge couplings present a more complicated case which we shall not deal with here. On the other hand, we know that the terms up to quadratic order with respect to the fields in the action - the kinetic and the mass terms - cannot give rise to non-decoupling effects by themselves since they do not generate interactions. To be systematic, this leaves the case of the dimensionful scalar cubic coupling within the renormalizable couplings in four dimensions. This case is of interest, since in some sense it lies in between the couplings that are known to give rise to non-decoupling effects and those that do not. Also, the dimensionless property of the couplings that give rise to non-decoupling effects is sometimes stressed [8]. It is of interest to find if the dimensionful couplings give rise to non-decoupling effects and if so, how the behavior non-decoupling

effects changes with the dimensionality of the coupling. Furthermore, these coupling arise naturally in some popular extensions of the Standard Model, such as the Supersymmetric 
Standard Model and it is important to understand what kind of physical effects the coupling produces.

In this note, we shall show that the cubic scalar coupling by itself generically does give rise to non-decoupling effects perturbatively and analyze the mass dependence of these effects. We find that the behavior is qualitatively different from those previously analyzed for the other couplings. While the need for investigating the effects of the scalar cubic coupling seems obvious, there is perhaps a reason why it has not been investigated by itself previously; the scalar cubic coupling tends to give rise to a classical instability in the absence of quartic couplings. However, this needs not be the case, as we shall see below.

\section{The model}

The model we consider has the following Lagrangian

$$
-\mathcal{L}=\left|\partial_{\mu} \phi\right|^{2}+\left|\partial_{\mu} \varphi_{L}\right|^{2}+m_{L}^{2}\left|\varphi_{L}\right|^{2}+\left|\partial_{\mu} \varphi_{R}\right|^{2}+m_{R}^{2}\left|\varphi_{R}\right|^{2}+\lambda\left(\varphi_{L}^{\dagger} \phi \varphi_{R}+\varphi_{R}^{\dagger} \phi^{\dagger} \varphi_{L}\right)
$$

$\phi, \varphi_{L}$ are scalar doublets and $\varphi_{R}$ is a scalar singlet; the reason for this choice of fields will become clear below. The model has no classical instability and has a flat direction along the $\phi$ direction. The symmetry of this model is $U(2)_{L} \times U(1)_{R}$ that acts on the fields of the theory thus

$$
\varphi_{L} \mapsto U_{L} \varphi_{L} \quad \varphi_{R} \mapsto e^{i \alpha} \varphi_{R} \quad \phi \mapsto U_{L} \phi e^{-i \alpha} \quad U \in \mathrm{U}(2), \alpha \in \mathbb{R}
$$

Some of the quartic couplings not included in the action allowed by symmetry are generated at the quantum level. Perturbatively, these quartic terms will have couplings which are of one loop order which only contribute at the two loop level and we shall not consider them below. In other words, we consider the region where the scalar cubic coupling is strong compared to other couplings and it is consistent to do so within perturbation theory. We shall elaborate more on this below.

This model may seem somewhat contrived. However, this model arises in the Supersymmetric Standard Model, wherein the scalar cubic coupling is the soft supersymmetry breaking parameter. There are additional couplings in the Supersymmetric Standard model, namely the electroweak gauge couplings - which are, of course weak - and the cubic superpotential (Yukawa) coupling, that both give rise to quartic some scalar couplings. The Yukawa coupling is weak for light fermion multiplets. In the Supersymmetric Standard Model, $\phi, \varphi_{L}$ and $\varphi_{R}$ correspond to one of the Higgs doublets, the supersymmetric partner of the fermion $S U(2)_{L}$ doublet and the singlet, respectively. We have chosen a model that is closely related to the Standard Model since it is relatively simple and it allows us to compute physical parameters with physical significance familiar to us. While the effects of the scalar cubic coupling has been included in studies of the Supersymmetric Standard Model, the effect of the cubic coupling by itself has not been cleanly delineated, to our knowledge. 
In the Standard Model, the model is gauged by changing the derivatives to the covariant derivatives. The covariant derivatives act on the fields as

$$
\begin{aligned}
D_{\mu} \varphi_{L} & \equiv\left(\partial_{\mu}-i g W_{\mu}^{a} \frac{\sigma^{a}}{2}-i g^{\prime} \frac{Y_{L}}{2} B_{\mu}\right) \varphi_{L}, \quad D_{\mu} \varphi_{R} \equiv\left(\partial_{\mu}-i g^{\prime} \frac{Y_{R}}{2} B_{\mu}\right) \varphi_{R} \\
D_{\mu} \phi & \equiv\left(\partial_{\mu}-i g W_{\mu}^{a} \frac{\sigma^{a}}{2}+i \frac{g^{\prime}}{2} B_{\mu}\right) \phi, \quad Y_{R}-Y_{L}=1
\end{aligned}
$$

The gauge couplings are considered as being weak in this model and quantum corrections due to the gauge sector will not be considered below.

The $\phi$ field has the vacuum expectation value along the flat direction, which breaks the symmetry to $U(1) \times U(1)$.

$$
\langle\phi\rangle=\left(\begin{array}{c}
v / \sqrt{2} \\
0
\end{array}\right), \quad \phi \equiv\left(\begin{array}{c}
1 / \sqrt{2}\left(v+\sigma+\chi^{0}\right) \\
\chi^{-}
\end{array}\right) .
$$

If desired, this may be uniquely arranged by including quartic couplings in the zero coupling limit analogous to the Prasad-Sommerfeld limit or using weak quartic couplings as in the Supersymmetric Standard Model. More concretely, we may consider adding a quartic interaction term $h\left(\phi^{\dagger} \phi-v^{2} / 2\right)^{2}$. Since this term only has to stabilize the vacuum expectation value against radiative corrections, the size of the coupling $h$ needs to be of only one loop order. Therefore, its contribution to the physical parameters is at the two loop level within perturbation theory. This statement may be made more precise if necessary: We could easily generalize the theory by replacing $\mathrm{SU}(2)_{L}$ by $\mathrm{SU}(N)_{L}$. In this case, we may control the size of the couplings systematically by using the large $N$ expansion. If we let $\lambda$ be of order $N^{-1 / 2}$ in the standard fashion, the corrections to the $\phi^{\dagger} \phi,\left(\phi^{\dagger} \phi\right)^{2}$ terms are of order $\mathcal{O}\left(N^{-1}\right), \mathcal{O}\left(N^{-2}\right)$ respectively so that the flat direction is preserved to leading order. Therefore, it is consistent to set a vacuum expectation value for $\phi$ as in (2.4). If desired, we may put in a coupling $h$ of order $N^{-b}(1<b<2)$, which does not produce any leading order radiative corrections but is enough to stabilize the vacuum.

When $v \neq 0$, the $\left(\varphi_{L}\right)_{1}$ and $\varphi_{R}$ are no longer mass eigenstates. The mass eigenstates $\tilde{\varphi}_{1}, \tilde{\varphi}_{2}$ are

$$
\left(\begin{array}{c}
\left(\varphi_{L}\right)_{1} \\
\varphi_{R}
\end{array}\right)=\left(\begin{array}{cc}
\cos \gamma & -\sin \gamma \\
\sin \gamma & \cos \gamma
\end{array}\right)\left(\begin{array}{c}
\tilde{\varphi}_{1} \\
\tilde{\varphi}_{2}
\end{array}\right)
$$

where

$$
\tan \gamma=\frac{1}{\sqrt{2} \lambda v}\left[-m_{L}^{2}+m_{R}^{2}+\sqrt{\left(m_{L}^{2}-m_{R}^{2}\right)^{2}+2 \lambda^{2} v^{2}}\right]
$$

The masses of $\tilde{\varphi}_{1}, \tilde{\varphi}_{2}$ are

$$
m_{1}^{2}, m_{2}^{2}=\frac{1}{2}\left[m_{L}^{2}+m_{R}^{2} \pm \sqrt{\left(m_{L}^{2}-m_{R}^{2}\right)^{2}+2 \lambda^{2} v^{2}}\right]
$$

The mass parameters satisfy the inequalities $0 \leq m_{1}^{2} \leq m_{L}^{2} \leq m_{2}^{2}$ and are otherwise independent. The particles in this model and their masses are $\phi[0], \tilde{\varphi}_{1}\left[m_{1}\right], \tilde{\varphi}_{2}\left[m_{2}\right],\left(\varphi_{L}\right)_{2}\left[m_{L}\right]$. 


\section{Contribution to the $\rho$ parameter}

To investigate the possibility of non-decoupling effects, we need to study a physically observable effect that is generated by quantum effects. Here, we first choose to study a wellknown physical parameter; the contribution of this model to the so called $\rho$ parameter, $\rho=$ $M_{W}^{2} /\left.\left(M_{Z}^{2} \cos ^{2} \theta_{W}\right)\right|_{p^{2}=0}$ [9] This parameter measures the asymmetry between the charged and the neutral gauge interactions at low energies, measured for instance in the ratio of low energy neutrino cross sections. We will obtain this contribution by two methods, first via the corrections to the scalar kinetic term and also through the current correlation functions. We show that they both give rise to the same contribution to leading order in the weak couplings, as they should.

\subsection{Quantum corrections to the scalar kinetic terms}

The contribution to $\rho$ is

$$
\rho=\left.\frac{Z_{+}}{Z_{0}}\right|_{\text {zero momentum }}=1+\left(Z_{+}-Z_{0}\right)_{\text {zero momentum }}+\mathcal{O}(Z-1)^{2}
$$

where $Z_{+}, Z_{0}$ are the coefficients of the kinetic terms for $\chi^{0}, \chi^{+}$. The $\rho$ parameter may be obtained from the ungauged scalar theory for the following reason [10] 11] [12]: The $\rho$ parameter may be thought of as the ratio of the masses of the gauge bosons. The mass terms for the gauge bosons may be obtained by minimally gauging the Nambu-Goldstone scalar kinetic terms. The coefficient for this kinetic term can hence be obtained within the scalar theory. This may be illustrated in the effective action if we consider the lowest dimension operator that contributes to the $\rho$ parameter; in this case, there is a unique operator that contributes to the scalar kinetic term, $\left(\phi^{\dagger} D_{\mu} \phi\right)^{2}$. Therefore, it may be obtained solely from the scalar theory without gauge interactions, to leading order.

To obtain the $Z$ factors, we compute the vacuum polarization graphs in fig. 1 .
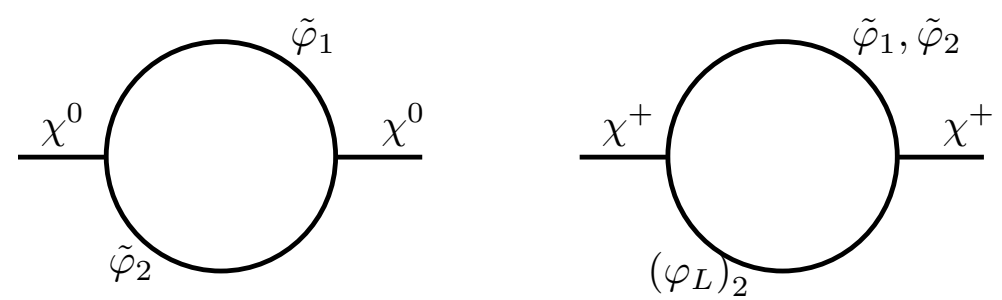

fig. 1 Contributions to the $\rho$ parameter computed within the scalar theory.

and obtain the self energies

$$
\pi_{0}\left(p^{2}\right)=\lambda^{2} I_{0}\left(p^{2} ; m_{1}^{2}, m_{2}^{2}\right), \quad \pi_{+}\left(p^{2}\right)=\lambda^{2}\left[\sin ^{2} \gamma I_{0}\left(p^{2} ; m_{1}^{2}, m_{L}^{2}\right)+\cos ^{2} \gamma I_{0}\left(p^{2} ; m_{2}^{2}, m_{L}^{2}\right)\right]
$$

where we defined

$$
I_{0}\left(p^{2} ; m_{a}^{2}, m_{b}^{2}\right) \equiv \int \frac{d^{4} k}{(2 \pi)^{4}} \frac{1}{\left((k+p)^{2}+m_{a}^{2}\right)\left(k^{2}+m_{b}^{2}\right)}
$$


After some manipulation, using $Z=1-d \pi / d p^{2}$, we obtain a compact formula

$$
\delta \rho=-\frac{\lambda^{4} v^{2}}{32 \pi^{2}} \int_{0}^{\infty} d x \frac{x\left(x^{2}-m_{1}^{2} m_{2}^{2}\right)}{\left(x+m_{1}^{2}\right)^{3}\left(x+m_{2}^{2}\right)^{3}\left(x+m_{L}^{2}\right)}
$$

This expression is finite both in the infrared and ultraviolet, as it should be. We explained how the correction corresponds to the $\delta \rho$ above. We stress here that this parameter is a physical parameter within the ungauged scalar theory; it describes the quantum generated asymmetry between the scalars at low energies, realized, for instance in scattering.

\subsection{Current correlation functions}

The $\rho$ parameter is the ratio of the strength of the charged current interactions to the neutral current interactions in the low energy limit. The correction to the $\rho$ parameter is

$$
\rho=\left.\frac{M_{W}^{2}}{M_{Z}^{2} \cos ^{2} \theta_{W}}\right|_{p^{2}=0}=1-\frac{\Pi_{W}}{M_{W}^{2}}+\frac{\Pi_{Z}}{M_{Z}^{2}}+\text { higher orders }
$$

Here we defined $\Pi_{W, Z}$ to be the the vacuum polarization of the gauge bosons. All the quantities in the above expression are evaluated at zero momentum. We compute the following current correlation functions fig. 2
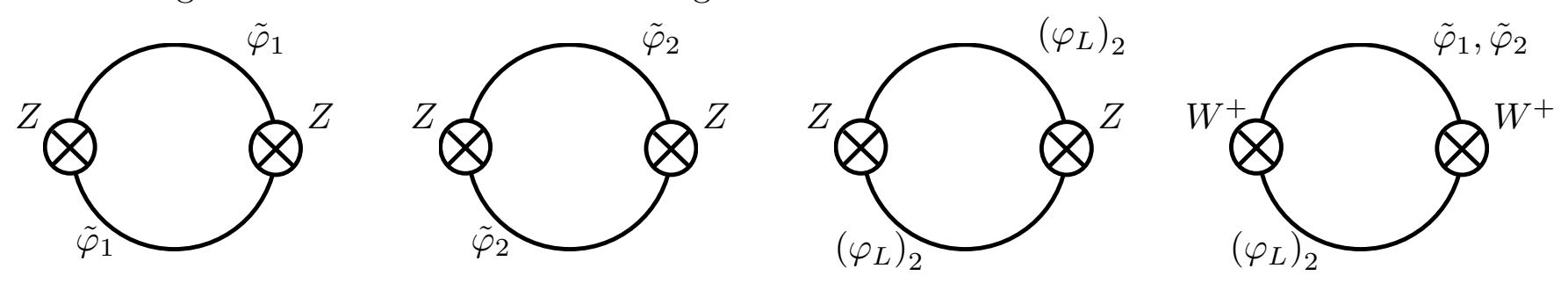

fig. 2 Contributions to the current correlation functions needed for obtaining the $\rho$ parameter.

to obtain

$$
\begin{aligned}
& \Pi_{Z}\left(p^{2}=0\right)=\frac{g^{2}}{4}\left[\cos ^{4} \gamma I_{1}\left(m_{1}^{2}, m_{1}^{2}\right)+\sin ^{4} \gamma I_{1}\left(m_{2}^{2}, m_{2}^{2}\right)+I_{1}\left(m_{L}^{2}, m_{L}^{2}\right)+2 \sin ^{2} \gamma \cos ^{2} \gamma I_{1}\left(m_{1}^{2}, m_{2}^{2}\right)\right] \\
& \Pi_{W}\left(p^{2}=0\right)=\frac{g^{2}}{2}\left[\cos ^{2} \gamma I_{1}\left(m_{1}^{2}, m_{L}^{2}\right)+\sin ^{2} \gamma I_{1}\left(m_{2}^{2}, m_{L}^{2}\right)\right]
\end{aligned}
$$

where we defined The seagull contributions cancel out in the expression for $\delta \rho$ and will not be discussed below. We obtain another compact formula for $\delta \rho$

$$
\delta \rho=\frac{\lambda^{4} v^{2}}{64 \pi^{2}} \int_{0}^{\infty} d x \frac{x^{2}}{\left(x+m_{1}^{2}\right)^{2}\left(x+m_{2}^{2}\right)^{2}\left(x+m_{L}^{2}\right)^{2}}
$$

After integration by parts, this expression is identical to the one obtained through the scalar kinetic terms, (3.4), as it should be. 


\subsection{Properties of $\delta \rho$}

$\delta \rho$ may be computed and expressed in terms of the particle masses $m_{1}^{2}, m_{2}^{2}, m_{L}^{2}$ as

$$
\begin{aligned}
\delta \rho= & \frac{1}{(4 \pi v)^{2}}\left[m_{L}^{2}+\frac{-4 m_{1}^{2} m_{2}^{2} m_{L}^{2}+\left(m_{1}^{2}+m_{2}^{2}\right)\left(m_{L}^{4}+m_{1}^{2} m_{2}^{2}\right)}{\left(m_{1}^{2}-m_{2}^{2}\right)^{2}}\right. \\
& \left.\quad-\frac{2 m_{1}^{2}\left(m_{2}^{2}-m_{L}^{2}\right)^{2}\left(m_{1}^{4}-m_{2}^{2} m_{L}^{2}\right) \log m_{1}^{2} / m_{L}^{2}}{\left(m_{1}^{2}-m_{2}^{2}\right)^{3}\left(m_{1}^{2}-m_{L}^{2}\right)}+\frac{2 m_{2}^{2}\left(m_{1}^{2}-m_{L}^{2}\right)^{2}\left(m_{2}^{4}-m_{1}^{2} m_{L}^{2}\right) \log m_{2}^{2} / m_{L}^{2}}{\left(m_{1}^{2}-m_{2}^{2}\right)^{3}\left(m_{2}^{2}-m_{L}^{2}\right)}\right]
\end{aligned}
$$

This formula is consistent with the previous results given in [13]. From this expression, it is clear that this is a non-decoupling effect since if we scale up all the masses without changing their ratios, the expression will scale up quadratically with the mass scale. This, of course, does not contradict the decoupling theorem since the coupling constant cannot be held fixed while all the masses are scaled up this way. We also find the following behavior for large $m_{1}^{2}$

$$
\delta \rho=\frac{1}{(4 \pi v)^{2}}\left[m_{2}^{2}+m_{L}^{2}-2 \frac{m_{2}^{2} m_{L}^{2}}{m_{2}^{2}-m_{L}^{2}} \log \frac{m_{2}^{2}}{m_{L}^{2}}+\mathcal{O}\left(\frac{m_{2}^{2}}{m_{1}^{2}}, \frac{m_{L}^{2}}{m_{1}^{2}}\right)\right]
$$

The non-decoupling effect arises because an increase in a particular mass parameter inevitably gives rise to an increase in the coupling. The effect is quadratic in the mass, which is similar to the Yukawa coupling, or to the case dimensionless coupling (1.1). (The Higgs contribution to the $\rho$ parameter is somewhat special since it is suppressed for symmetry reasons [14].) If we analyze the behavior of the non-decoupling effect with respect to the coupling, it is linear with respect to the coupling. This is a milder dependence than the contribution from a dimensionless coupling where the behavior is generically quadratic in the coupling to leading order. In this sense, we see that the behavior of the non-decoupling effect due to a dimensionful coupling lies somewhere in "between" that of the dimensionless coupling and the free case.

\section{4. $S$ parameter}

In this section, we compute the contribution to the so-called $S$ parameter [15] and the contribution to it measured using the longitudinal modes of the gauge boson - the Nambu-Goldstone bosons - which we shall call $\tilde{S}$ [5].

\section{1. $\tilde{S}$}

$\tilde{S}$ parameter is the amount of mixing between $W^{3}$ and $B$ gauge bosons measured through their longitudinal modes. It may be calculated within the scalar theory as

$$
\tilde{S} \equiv-\left.2 \pi v^{2} \frac{d}{d\left(p^{2}\right)} Z_{0}\left(p^{2}\right)\right|_{p^{2}=0}
$$

This parameter measures how the effective coupling changes with the momentum at zero energies and is a physical parameter within the scalar theory. 
From the expression for the scalar self energy in (3.2), we obtain

$$
\begin{aligned}
\tilde{S} & =\lambda^{2} \int_{0}^{\infty} d x \frac{x}{\left(x+m_{1}^{2}\right)^{3}\left(x+m_{2}\right)}\left[\frac{m_{1}^{2}}{x+m_{1}^{2}}-\frac{2 x m_{1}^{2}}{\left(x+m_{1}^{2}\right)^{2}}\right] \\
& =-\frac{1}{12 \pi} \frac{\left(m_{1}^{2}-m_{L}^{2}\right)\left(m_{2}^{2}-m_{L}^{2}\right)\left[m_{1}^{6}-m_{2}^{6}+9\left(m_{1}^{4} m_{2}^{2}-m_{1}^{2} m_{2}^{4}\right)-6 m_{1}^{2} m_{2}^{2}\left(m_{1}^{2}+m_{2}^{2}\right) \log m_{1}^{2} / m_{2}^{2}\right]}{\left(m_{1}^{2}-m_{2}^{2}\right)^{5}}
\end{aligned}
$$

Expressed this way, the $\tilde{S}$ parameter is again clearly a non-decoupling effect. If we increase the masses while keeping their ratios fixed, the parameter stays constant, so that this is again a non-decoupling effect. $\tilde{S}$ has a milder behavior with respect to the particle mass when compared with the $\rho$ parameter. Especially for large $m_{1}^{2}$, it has a simple behavior

$$
\tilde{S}=\frac{1}{12 \pi} \frac{m_{L}^{2}-m_{2}^{2}}{m_{1}^{2}}\left[1+\mathcal{O}\left(\frac{m_{2}^{2}}{m_{1}^{2}}, \frac{m_{L}^{2}}{m_{1}^{2}}\right)\right]
$$

If we keep the mass ratio between $m_{1}^{2}$ and $m_{2}^{2}$ and increase the particle masses, the $\tilde{S}$ parameter remains constant. This is reminiscent of the behavior of the contribution to the $S$ parameter from the Yukawa coupling in the Standard Model, wherein the part that remains constant with the particle mass is generated by the longitudinal modes of the gauge bosons, as it was here.

\section{2. $S$ parameter}

The so-called $S$ parameter [15] may be defined as the amount of mixing between $W_{3}$ and $B$ as in

$$
S \equiv-\frac{16 \pi}{g g^{\prime}} \frac{d}{d p^{2}} \Pi^{W^{3} B}(0)
$$

This may be computed from the graphs in fig. 3
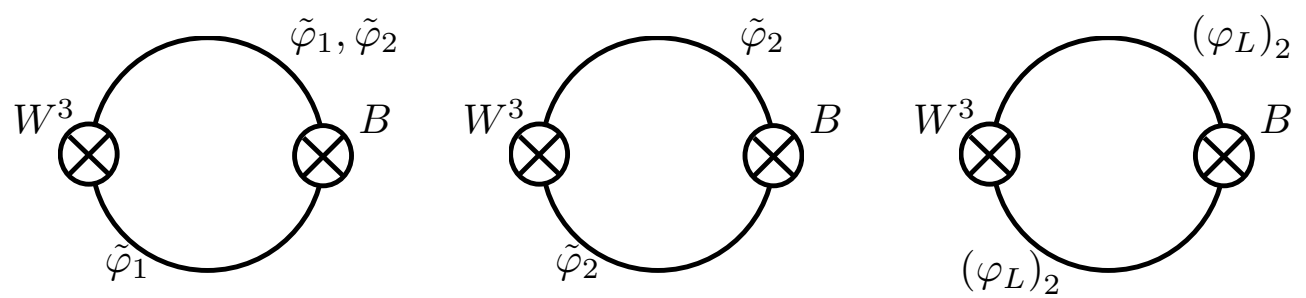

fig. 3 Graphs contributing to the $S$ parameter

to obtain

$$
\begin{gathered}
\Pi_{\mu \nu}^{W^{3} B}=\frac{g g^{\prime}}{12}\left[\cos ^{2} \gamma\left(1+3 \sin ^{2} \gamma\right) K_{\mu \nu}\left(p^{2} ; m_{1}^{2}, m_{1}^{2}\right)+\sin ^{2} \gamma\left(1+3 \cos ^{2} \gamma\right) K_{\mu \nu}\left(p^{2} ; m_{2}^{2}, m_{2}^{2}\right)\right. \\
\left.-K_{\mu \nu}\left(p^{2} ; m_{L}^{2}, m_{L}^{2}\right)-6 \cos ^{2} \gamma \sin ^{2} \gamma K_{\mu \nu}\left(p^{2} ; m_{1}^{2}, m_{2}^{2}\right)\right]
\end{gathered}
$$

where

$$
K_{\mu \nu}\left(p^{2} ; a, b\right) \equiv \int \frac{d^{4} k}{(2 \pi)^{4}} \frac{(2 k+p)_{\mu}(2 k+p)_{\nu}}{\left(k^{2}+a\right)\left((k+p)^{2}+b\right)}
$$


After some computation, we obtain the $S$ parameter as

$$
\begin{aligned}
S=\frac{1}{12 \pi} & \left\{\frac { ( m _ { 1 } ^ { 2 } - m _ { L } ^ { 2 } ) ( m _ { 2 } ^ { 2 } - m _ { L } ^ { 2 } ) } { ( m _ { 1 } ^ { 2 } - m _ { 2 } ^ { 2 } ) ^ { 5 } } \left[\frac{5}{3}\left(m_{1}^{6}-m_{2}^{6}\right)-9 m_{1}^{2} m_{2}^{2}\left(m_{1}^{2}-m_{2}^{2}\right)\right.\right. \\
& \left.+\left(-m_{1}^{6}-m_{2}^{6}+3 m_{1}^{2} m_{2}^{2}\left(m_{1}^{2}+m_{2}^{2}\right)\right) \log \frac{m_{1}^{2}}{m_{2}^{2}}\right] \\
& \left.-Y_{L} \frac{-\left(m_{2}^{2}-m_{L}^{2}\right) \log m_{1}^{2} / m_{L}^{2}+\left(m_{1}^{2}-m_{L}^{2}\right) \log m_{2}^{2} / m_{L}^{2}}{m_{1}^{2}-m_{2}^{2}}\right\}
\end{aligned}
$$

We compare $\tilde{S}$ and $S$ and obtain the difference as

$$
\begin{aligned}
S-\tilde{S}= & \frac{1}{12 \pi}\left\{\frac{\left(m_{1}^{2}-m_{L}^{2}\right)\left(m_{2}^{2}-m_{L}^{2}\right)\left[8 / 3\left(m_{1}^{6}-m_{2}^{6}\right)+\left(m_{1}^{2}+m_{2}^{2}\right)^{3} \log m_{1}^{2} / m_{2}^{2}\right]}{\left(m_{1}^{2}-m_{2}^{2}\right)^{5}}\right. \\
& \left.-Y_{L} \frac{-\left(m_{2}^{2}-m_{L}^{2}\right) \log m_{1}^{2} / m_{L}^{2}+\left(m_{1}^{2}-m_{L}^{2}\right) \log m_{2}^{2} / m_{L}^{2}}{m_{1}^{2}-m_{2}^{2}}\right\}
\end{aligned}
$$

The difference, unlike the case of the Yukawa coupling for the fermions, is not proportional to $Y_{L}$ but rather to both $Y_{L, R}$. For large $m_{1}^{2}$, the difference in proportional to the $\mathrm{U}(1)_{Y}$ hypercharge $Y_{L}$, in a manner reminiscent of the Yukawa coupling case.

$$
S-\tilde{S}=-\frac{1}{12 \pi} Y_{L} \log \frac{m_{2}^{2}}{m_{L}^{2}}+\mathcal{O}\left(\frac{m_{2}^{2}}{m_{1}^{2}}, \frac{m_{L}^{2}}{m_{1}^{2}}\right)
$$

In the case of the $\rho$ parameter, the longitudinal contribution and the full contribution were identical, as is required by the gauge symmetry. For the $S$ parameter we find that it is not so [11] [12]; the difference may be understood as follows. Considering the effect from the effective action, the lowest dimension operator that contributes to the $S$ parameter has dimension eight. At dimension eight, only one operator, $\left(\phi^{\dagger} D_{\mu} D_{\nu} \phi\right)^{2}$ contributes to the $\tilde{S}$ parameter. There is an additional operator $\left(\phi^{\dagger} W_{\mu \nu} \phi\right)\left(\phi^{\dagger} B_{\mu \nu} \phi\right)$ that contributes to $S$, that does not involve the scalar kinetic term. It does not seem possible to determine this coefficient within the scalar theory.

\section{Discussion}

In this note, we showed how the cubic coupling in the scalar theory may give rise to non-decoupling effects perturbatively and analyzed these effects, such as their mass dependence. It is widely known that the dimensionless couplings can give rise to nondecoupling effects and the dimensionless nature of the couplings have sometimes been stressed. It is on the other hand clear that the mass (quadratic) terms by themselves do not give rise to non-decoupling effects. We have clearly shown that the dimensionful scalar cubic coupling does give rise to non-decoupling effects by itself. This basically completes the picture as to which renormalizable couplings may give rise to non-decoupling effects and which do not. 
The contribution to the $\rho$ and the $S$ parameters from the scalar trilinear coupling, somewhat surprisingly, turn out to be substantially more complicated than those for the Yukawa coupling or the Higgs coupling. When reinterpreted in terms of the coupling constant, the $\delta \rho$ contribution is linear in the coupling to leading order. This is a feature not observed in the non-decoupling effects due to dimensionless couplings. Roughly speaking, the strength of the contribution is somewhat weaker than that from a dimensionless coupling.

The gauge bosons derive their masses from their couplings to the Nambu-Goldstone bosons. Due to gauge invariance, one might expect that the non-decoupling effects might be obtained by examining just the Nambu-Goldstone dynamics. If this were possible, it would be instrumental in analyzing the systematics of these effects, since we may summarize the contributions within the scalar theory. Also, if we only had to perform scalar computations, the work would be facilitated substantially. This is somewhat similar in philosophy to the so-called "equivalence theorems" used in high energy scattering of $W$ bosons. [16] For leading contributions to the $\rho$ parameter, this is indeed the case, as was shown some time ago [10]. In general, this clearly can not be true since the scalar theory knows nothing about the $\mathrm{U}(1)_{Y}$ hypercharge. Even if we were to ignore the hypercharge, the effect still needs not be computable by examining only the longitudinal modes in general [11]. We indeed find that the contribution to the $S$ parameter is different from the contribution obtained from the longitudinal modes of the gauge bosons for which we gave reasons as to why this is so. In our example, the difference is not proportional to $Y_{L}$ even at leading order, which seems to be a rather novel feature.

Finally, it may be prudent to reflect on what the non-decoupling effects are in a precise sense: In some theories, a mass parameter can not be generated by a quadratic mass term in the Lagrangian for symmetry reasons. In such cases, it may be possible to generate the mass term through a coupling constant and a vacuum expectation value of a field. If such cases, the increase in the mass parameter necessarily entails stronger coupling and we evade the assumptions of the decoupling theorem. This may lead to quantum effects that do not decrease with the increase in this mass parameter while this is not inevitable. In the Standard Model, the fermion and the Higgs mass terms are forbidden due to gauge symmetry. In the case we analyzed, the symmetry reasons - which can be gauge symmetry, just as in the Standard Model - forbid the appearance of certain mass parameters, more specifically, the mixing between $\left(\varphi_{L}\right)_{1}$ and $\varphi_{R}$. This mixing will necessarily give rise to certain non-decoupling effects.

Acknowledgments: We would like to thank Santiago Peris for invaluable discussions. This work was supported in part by the Grant-in-Aid from the Ministry of Education, Science, Sports and Culture and grants from Keio University. 


\section{References}

[1] T. Appelquist, J. Carazzone, Phys. Rev. 11 (1975) 2856

[2] Review of particle properties, Phys. Rev. D45 (1992) S1, Phys. Lett. 239B (1990) 1 and references therein.

[3] CDF Collaboration (F. Abe et al.), Phys. Rev. Lett. 74 (1995) 2626

D0 Collaboration (S. Abachi et al.), Phys. Rev. Lett. 74 (1995) 2632

[4] K. Aoki, S. Peris, Zeit. Phys. C61 (1994) 303

K. Aoki, S. Peris, Phys. Lett. B335 (1994) 470

[5] K. Aoki, S. Peris, Phys. Rev. Lett. 70 (1993) 1743

[6] S. Cortese, E. Pallante, R. Petronzio, Phys. Lett. 301B (1993) 203

[7] Review of particle properties, Phys. Rev. D54 (1996) 1, and references therein.

[8] See for example, J.C. Collins, "Renormalization", Cambridge University Press (1984), Chapter 8.

[9] M. Veltman, Nucl. Phys. B123 (1977) 89

[10] R.S. Lytel, Phys. Rev. 22D (1980) 505

A.C. Longhitano, Nucl. Phys. B188 (1981) 118

[11] R.D. Peccei, S. Peris Phys. Rev. D44 (1991) 809

[12] F. Feruglio, A. Masiero, L. Maiani, Nucl. Phys. B387 (1992) 523

[13] C.S. Lim, T. Inami, N. Sakai, Phys. Rev. D29 (1984) 1488

[14] M. Veltman, Acta Phys. Pol. B8(1977); Phys. Lett. 70B (1977) 253

M.B. Einhorn, J. Wudka, Phys. Rev. 39D (1989) 39

[15] B.W. Lynn, M. Peskin and R.G. Stuart in "Physics at LEP", eds. J. Ellis and R.D. Peccei (1986)

M. Peskin, T. Takeuchi, Phys. Rev. Lett. 65 (1990) 964

D.C. Kennedy, P. Langacker Phys. Rev. Lett. 65 (1990) 2967

G. Altarelli, R. Barbieri Phys. Lett. 253B (1991) 161

[16] J.S. Bell, Nucl. Phys. B60 (1973) 428

C.H. Lewellyn Smith, Phys. Lett. 46B (1973) 233

J.M. Cornwall, D.N. Levin, G. Tiktopoulos, Phys. Rev. Lett. 30 (1973) 1268

M. Chanowitz, M.K. Gaillard, Phys. Lett. 142B (1984) 85 